\title{
NEW MODELS FOR THE CONVECTIVE FLUX IN STELLAR
}

\section{ATMOSPHERES}

\author{
F. KUPKA \\ Institute for Astronomy, Univ. of Vienna, \\ Türkenschanzstr. 17, A-1180 Vienna, Austria
}

\begin{abstract}
Over the past decades various forms of the mixing length theory (MLT) have been used to describe convection in stellar atmospheres. Recent advances in turbulence theory now allow for major improvements in modelling thermal convection. We review several models for convection which have been derived from turbulence theory, and describe one of them, the "CM model", in detail. The CM model has been used in several stellar evolution and helioseismology codes during the last four years and has now been applied to model atmospheres. An overwiew comparing stellar atmosphere models based on the CM formulation with its MLT predecessors indicates improvements on model atmospheres for $\mathrm{A}$ and $\mathrm{F}$ stars.
\end{abstract}

\section{Introduction}

Even stellar model atmospheres that neglect magnetic fields, rotation, mass loss, etc. cannot be constructed from first principles alone, since the resulting equations can neither be solved with numerical nor analytical methods currently available. In order to keep the basic equations of hydrodynamics and radiative transfer managable for parameter ranges encountered in stars, they still have to be simplified to allow at least numerical solution.

In most numerical studies of stellar convection, radiative transfer is treated in the diffusion approximation, see for example Xie and Toomre (1993) and references cited therein. Intended primarily to study basic properties of stellar convection, these codes are not applicable to study the surface of stars or to construct model atmospheres. Because of the low viscosity of the stellar gas (e.g. Edmonds (1957) or Massaguer (1990)), energy transport and energy dissipation occure at very different scales which currently cannot be resolved at the same time. Thus the common approach is to sim- 
ulate only the large scales explicitely (large eddy simulations), whereas the unresolved ones have to be treated with some form of subgrid modelling, as described in Canuto (1994).

To study solar granulation, Nordlund and Dravins (1990) have used a similar type of code, but included a more general approximation to solve the equation of non-grey radiative transfer. Although this and similar codes have been used to construct model atmospheres, their large computational demands, their still simplified treatment of radiative transfer and the nontrivial problem of averaging physical quantities to compute synthetic spectra are hindering them in replacing classical model atmospheres.

As a consequence, the most widely used type of model atmosphere is still an average not only in energy (frequency intervals used in integrating the equation of radiative transfer), but also in time and space (static, onedimensional models). The only remnant of the dynamic properties of the gas retained by these models is energy transport by thermal convection, the convective flux. This quantity has been modelled by means of the mixing length theory (MLT) for several decades. In the following sections we intend to review more advanced modelling of turbulent convection and discuss the application of one of them, the CM model (Canuto and Mazzitelli (1991, 1992), hereafter CM91 \& CM92), to classical model atmospheres.

\section{Turbulent convection}

Turbulent convection deals with turbulence generated by mean temperature gradients. The basic concept of modern turbulence theory is the ensemble average: The physical quantities describing the system (velocity, temperature, pressure, density) are split into average and fluctuating parts. The resulting set of equations has to be "closed" to contain as many equations as unknowns. Several different closures have already been used to derive models of turbulent convection in stars.

One is the Reynolds stress approach. The idea behind this method is to write the equations of the fluctuating components or "first order moments" (temperature, velocity, etc.) using "second order moments" (algebraic products of the various fluctuating quantities). One of these second order moments, $\overline{w \theta}$, is the convective flux: $F_{c}=c_{p} \rho \overline{w \theta}$, where $w$ is the velocity fluctuation (convective velocity) in the direction against the temperature gradient and $\theta$ the corresponding temperature fluctuation. For the second order moments equations have to be derived, which entail third order moments. The closure can be done for forth order moments, which are written in terms of second order moments (thus the name second order closure or SOC). Xiong (1985) and Canuto (1993) have used the Reynolds stress approach to derive models for stellar turbulent convection. The main strength of this 
approach is the inclusion of non-locality and pressure anisotropy, which play a crucial role near the "boundary" between stable and unstable layers and for describing overshooting. However, the assumption of incompressibility is at the heart of the Reynolds stress approach and the incorporation of additional physics (magnetic fields, etc.) is quite difficult.

A completely different approach is the two-point closure. The main idea of this method is to compute the energy spectral function $E(k)$, which gives the average turbulent kinetic energy per mass for eddies ("convective elements") of all sizes. Solving the two-point closure equations by various formalisms, as described in CM91, means to describe how large and small eddies interact with each other. The turbulent kinetic energy spectrum $E(k)$ is used to compute the convective flux (see, e.g., Yamaguchi (1963)),

$$
F_{c}=\frac{c_{p} \rho}{g \alpha} \int_{0}^{\infty} 2 E(k)\left[n_{s}(k)+\nu k^{2}\right] d k,
$$

with $\alpha$ being the thermal expansion coefficient, $\nu$ the kinematic viscosity, and the meaning of the other symbols being standard, provided that a "growth rate" $n_{s}(k)$ is supplied to represent the energy injected into the system to keep turbulence from decaying. Thus effects on the convective flux caused by magnetic fields, rotation, radiative losses, a radiative layer at the top of the convective zone (instead of a fixed plate) etc. can be included in the convection model. However, the assumption of homogeneity is at the heart of all two-point closure formalisms, and because convective flows near the boundary to stable regions and in overshooting regions are inhomogenous, overshooting cannot be modelled by them.

\section{Comparing MLT and the CM model}

The MLT can be recovered from modern turbulence theories. In the twopoint closure framework, the MLT expression for the convective flux is obtained by assuming that the energy spectrum $E(k)$ is a delta function peaked at the wave number $k_{0}$ of the maximum of the "real" energy distribution. In CM91 a two-point closure model assuming incompressibility was chosen to compute the turbulent energy spectrum $E(k)$ in the stationary limit. Two plots given in their paper are shown in Figure 1. In the left plot the energy spectrum of their convection model is compared with the MLT's "spectrum" for a Prandtl number $\sigma$ and a value of $S=R a \sigma$ (the product of both Rayleigh and Prandtl number) applicable to stellar astrophysics. Although the Prandtl number is higher than that one usually encountered in stellar astrophysics, the spectrum does not change significantly for lower values of $\sigma$ (because 0.001 represents a fluid with already very low viscosity), whereas for higher values of $\sigma$ the shape is quite different (plot at the 

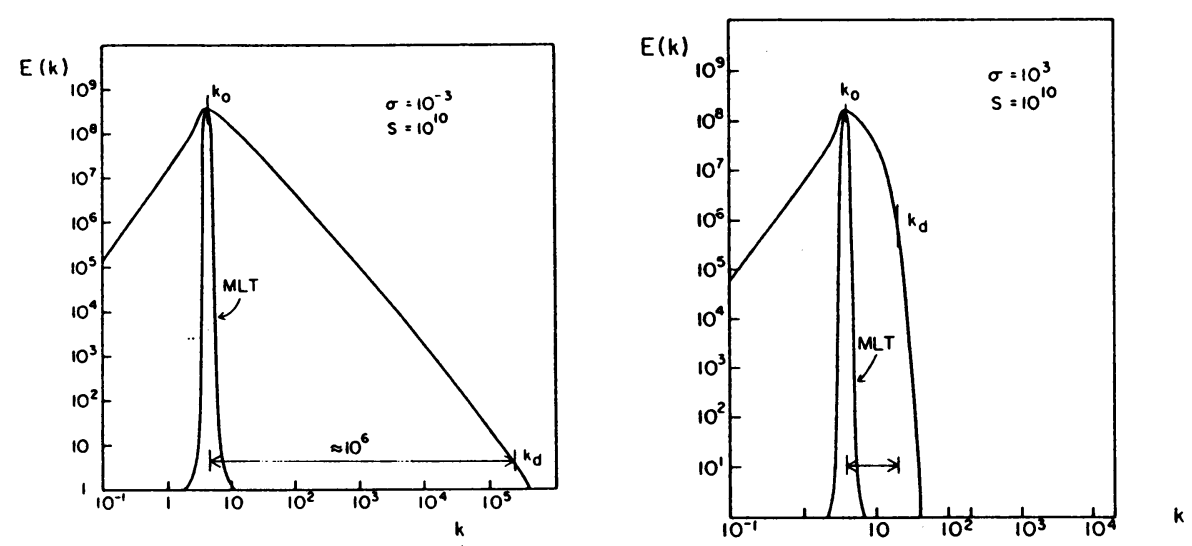

Figure 1. Turbulence energy spectrum for fluids of different viscosity: Low $\sigma$ as in stars (left plot) and high $\sigma$ as for oil (right plot). The position of $k_{d}$ indicates the wavenumber of the onset of dissipation. See text for details.

right hand side). As seen from Figure 1, the MLT is a poor representation of the energy spectrum relevant to convection in stars, which finally results in an underestimation of the convective flux in the high efficiency regime and an overestimation in the low efficiency regime of convection (a detailed explanation will be given in Canuto and Kupka (1996)).

Canuto and Mazzitelli used a general formula for the convective flux,

$$
F_{c}=K T H_{p}^{-1}\left(\nabla-\nabla_{a d}\right) \Phi\left(\nabla-\nabla_{a d}, A\right),
$$

where $K=4 a c T^{3} /(3 \kappa \rho)$ is the radiative conductivity, $\Phi$ is the ratio of turbulent to radiative conductivity, and the meanings of the other symbols being standard. Furthermore (see CM91 and CM92),'

$$
\Sigma=4 A^{2}\left(\nabla-\nabla_{a d}\right), \quad A=\frac{Q^{1 / 2} c_{p} \rho^{2} \kappa \Lambda^{2}}{12 a c T^{3}} \sqrt{\frac{g}{2 H_{p}}},
$$

where $\Lambda$ is the mixing length and $Q$ represents the variable average molecular weight. The quantity $\Sigma$ is related to $S$ by the simple relation $\Sigma=\frac{2}{81} S$. The standard form of MLT (see CM91) can be written as

$$
\Phi^{\mathrm{MLT}}=\frac{9}{8} \Sigma^{-1}[\sqrt{1+\Sigma}-1]^{3} .
$$

For an (almost) inviscid fluid a convective system depends on just one dimensionless number independant of viscosity, for example $S$ itself. Hence, in CM91, the convective flux for low Prandtl numbers as given by the turbulence model was computed as a function of $S$, which can be written in terms of local physical quantities. The CM form for $\Phi$ is

$$
\Phi=a_{1} \Sigma^{m}\left[\left(1+a_{2} \Sigma\right)^{n}-1\right]^{p}, \quad \text { where }
$$




$$
a_{1}=24.868, a_{2}=0.097666, \quad m=0.14972, n=0.18931, p=1.8503 \text {. }
$$

The new function $\Phi^{\mathrm{CM}}$, consisting of (5) and (6) to replace the MLT expression (4), is the first ingredient of the "CM model". It yields about 10 times more flux than the MLT expression for high values of $S$ (high convective efficiency) and only one tenth for low ones. The asymptotic behaviour of both is equal, a result obtainable from dimensional analysis.

As $\Phi$ is written entirely in local variables, the expression naturally depends on a characteristic length (equivalent to the mixing length for the MLT expression), which cannot be provided by the formalism itself. Taking into account that the assumption of incompressibility provides no natural unit of length and that eddies near the boundary of the convective zone are smaller than in the middle of the same (stacking), CM91 proposed $\Lambda=z$, with $z$ being the distance to the nearest stable layer. The new flux and the prescription for $\Lambda$ have subsequently been called the "CM model".

The CM model with its parameter free prescription of $\Lambda$ proved to work better than or at least as well as MLT in various astrophysical applications: helioseismology, stellar structure and evolution, see for instance Stothers and Chin (1995). The reason for this improvement is the consideration of a realistic model of the distribution of energy among eddies of various sizes present in a convection zone instead of MLT's one-eddy approximation.

\section{Implementation of the CM model into ATLAS9}

The simple algebraic form of $\Phi^{\mathrm{CM}}$ makes the CM model easily applicable to classical model atmospheres. We have selected the ATLAS9 code by Kurucz (1993) to investigate the effect of the new convection model on atmospheres with plane-parallel geometry. ATLAS9 computes flux constant model atmospheres (the flux consisting of a radiative and a convective part) with an opacity distribution function treatment of line blanketing. The following changes had to be made to the code:

- A new temperature correction, based on a general form of $\Phi$,

- computation of $\Lambda=z$ to replace the MLT's prescription of $\Lambda$ as a fraction of the pressure scale height $H_{p}$,

- and a replacement of the correction for optically thin layers of $\Phi$ in ATLAS9, which had been specifically designed for MLT.

Details on the implementation will be given in Canuto and Kupka (1996).

\section{Results}

The modified ATLAS9 was used to compute grids of model atmospheres for main sequence stars and giants from $T_{\text {eff }}=3500 \mathrm{~K}$ to the cease of convective instability in stellar atmospheres at about $10000 \mathrm{~K}$. Details on the 
results will be presented in Canuto and Kupka (1996) as well as subsequent publications. An interesting example is the effect of lower convective efficiency in model atmospheres for $\mathrm{A}-\mathrm{F}$ main sequence stars. There are two reasons for this phenomenon: $\Phi^{\mathrm{CM}} \approx 0.1 \times \Phi^{\mathrm{MLT}}$ for small $S$, which naturally happens where the gas becomes stable against local perturbations and in addition, the prescription of $\Lambda=z$ causes $\Lambda \rightarrow 0$ at the top of the convection zone, whereas the MLT prescription of $\Lambda$ being a fraction of $H_{p}$ causes $\Lambda \rightarrow$ constant. This enhances the tendency of $\Phi^{\mathrm{CM}}$ to predict $F_{\mathrm{c}}$ to be small against the total flux near the boundary to the top (and bottom) of the convection zone. Consequently, the CM model produces a temperature gradient close to the radiative one in that region, which is the reason for the most important differences of $\mathrm{CM}$ based model atmospheres for A-F main sequence stars to their MLT counterparts:

- The onset of efficient convection (which carries, e.g., $50 \%$ of the total flux) is appearing for $T_{\text {eff }}$ about $1000 \mathrm{~K}$ lower than in the case of MLT (the exact numerical criterion of the relative amount of convective flux is not important for the size of that shift),

- for given (uncalibrated). colors, $T_{\text {eff }}$ can be less by more than $100 \mathrm{~K}$, the surface gravity $\log g$ more by up to 0.1 ,

- computed equivalent widths of lines formed in deep photospheric regions can differ up to a few percent from that one obtained through an MLT based model atmosphere,

- and also cool A stars have essentially radiative temperature gradients.

The left plot of Figure 2 displays the T- $\tau$ relation for both CM and MLT models for $T_{\text {eff }}=7900 \mathrm{~K}, \log g=4.2$, and solar metallicity, which can be used as a first (approximative) model for the Ap star $\alpha$ Cir. An abundance analysis of this object is published in Kupka et al. (1995). The small "dip" of the MLT model has disappeared in the CM temperature gradient, which causes slightly enhanced equivalent widths for some lines, if the computation is based on the CM model (see Figure 3). The large difference between both gradients in Figure 2 is already below the photosphere.

A different example is the solar model atmosphere. For the sun the onset of convective instability is located deeper inside than for $\alpha \mathrm{Cir}$ (in terms of the Rosseland optical depth). The right plot of Figure 2 displays a solar model atmosphere for $T_{\text {eff }}=5777 \mathrm{~K}$ and $\log g=4.4377$, the same values as used by Kurucz (1993). Compared to their $\alpha$ Cir counterparts, the CM and MLT temperature gradients start to diverge at greater optical depth. The different location of the convection zone in the sun has important consequences on the interpretation of phenomena related to convection. In the solar atmosphere we can observe the top of the convection zone and the overshooting region. The first one is most sensitive to the particular choice of $\Lambda$ and the latter one cannot be modelled by CM and MLT. In 

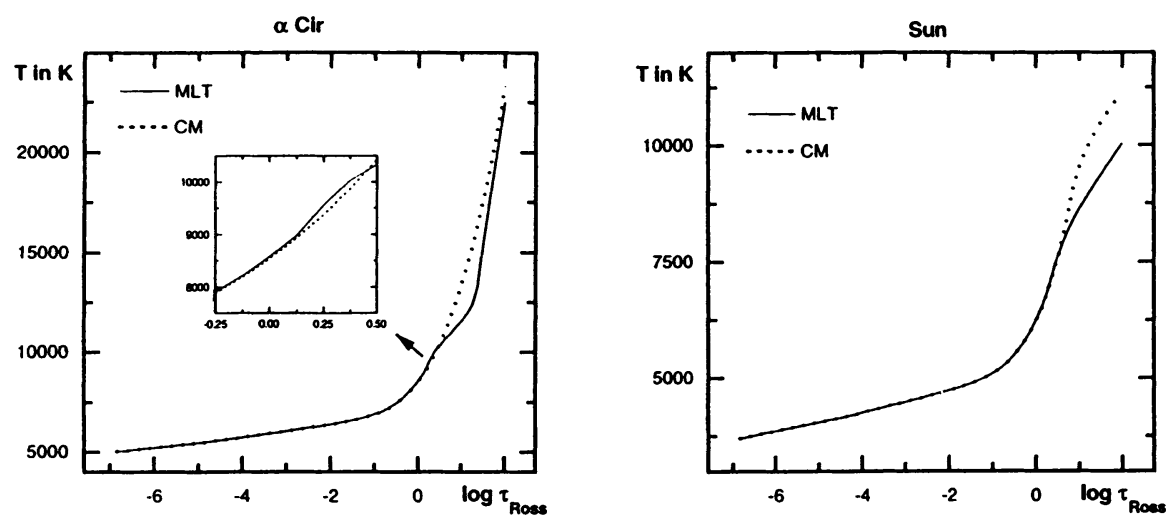

Figure 2. Temperature as a function of Rosseland optical depth for $\alpha$ Cir (left plot) and for the sun (right plot) for CM and MLT. The "dip" mentioned in the text is magnified.

addition, this region is known to be influenced by local magnetic fields. Hence, great care is recommended, when convection models are "calibrated" with solar photosphere observations. One particular example is the intensity at the central disk of the sun. Comparing observations by Neckel and Labs (1984) with intensities from model atmospheres, significant differences can be noticed in the region around $460 \mathrm{~nm}$. As will be discussed in Canuto and Kupka (1996), the MLT based intensities give a better fit in that region (due to the choice of $\Lambda$ ). However, for the blue wing of $\mathrm{H}_{\gamma}($ at $434 \mathrm{~nm}$ ) the $\mathrm{CM}$ based intensities give a better fit. Thus the comparison of computed and observed intensities allows no conclusions on the convection models.

\section{Conclusions}

The implementation of the CM model into ATLAS9 has revealed that classical model atmospheres can be improved by convection models based on modern turbulence theory. The convection zone in late A stars with high convective fluxes and velocities, as predicted by MLT, has vanished in CM based models. This convection zone has sometimes been considered as artifactual, due to the high efficiencies and velocities in a rather small layer. More important is the fact that the actual convection zone is clearly smaller than one pressure scale height whereas the mixing length has been taken larger than $H_{p}$. In the CM model this unphysical prescription has disappeared. For the solar case the improvements are not so obvious as the changes take place in regions accessible to helioseismology rather than direct observations. In that sense, the CM model has to be seen as the first logical successor to MLT in a sequence of improved convection models based on turbulence theory which may be used to develop "classical model atmospheres" beyond some of their current limitations. 


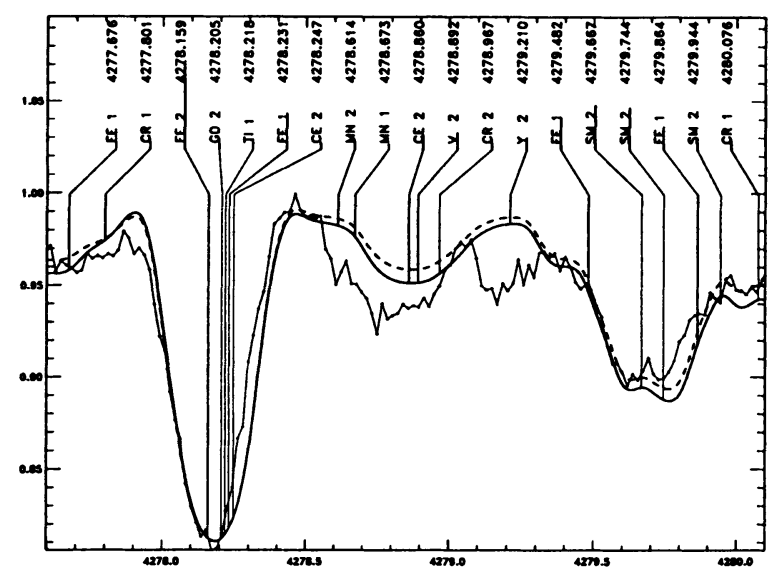

Figure 3. The CM model (thick line) indicates slightly less peculiar abundances for $\alpha$ Cir than an MLT based model atmosphere (dashed line). Average abundances have been used to compare with observations (thin line). Vertical bars indicate contributions to blends.

\section{Acknowledgements}

This research was done within the working group Asteroseismology-AMS (Fonds zur Förderung der wiss. Forschung, project S-7303 AST). Calculations were performed with an AXP-3000/600, funded through an DECEERP project (STARPULS). The author is grateful to V.M. Canuto for helpful discussions on turbulence theory and the CM model and to R.L. Kurucz for permitting usage of ATLAS9 and opacity data from CDROM.

\section{References}

Canuto, V.M. (1993), ApJ, 416, 331

Canuto, V.M. (1994), ApJ, 428, 729

Canuto, V.M. and Mazzitelli, I. (1991), ApJ, 370, 295

Canuto, V.M. and Mazzitelli, I. (1992), ApJ, 389, 724

Canuto, V.M. and Kupka, F. (1996), in preparation.

Edmonds, F.N. (1957), ApJ, 125, 535

Kupka, F., Ryabchikova, T.A., Mathys, G., Weiss, W.W., Kuschnig, R. and Rogl, J. (1995), $A \mathscr{E} A$, in print

Kurucz, R.L. (1993), CDROM13: ATLAS9. SAO, Harvard, Cambridge.

Massaguer, J.M. (1990), Inside the Sun, ed. G. Berthomieu and M. Cribier. Oxford University Press, Oxford.

Neckel, H. and Labs, D. (1984), Solar Physics, 90, 205

Nordlund, $\AA$ and Dravins, D. (1990), $A \& A, 228,155$

Stothers, R.B. and Chin, C.W. (1995), $A p J, 440,297$

$\mathrm{Xie}, \mathrm{X}$. and Toomre, J. (1993), $A p J, 405,747$

Xiong, D.R. (1985), $A \mathscr{E} A, \mathbf{1 5 0}, 133$

Yamaguchi, S. (1963), PASJ, 15, 412 